\title{
Diagnostic Accuracy of Doppler Ultrasonography in Predicting Presence of Esophageal Varices in Patients with Hepatitis-C Induced Cirrhosis
}

\author{
Ali Mansoor1, Rabia Shaukat², Aamer Nadeem Chaudhary² and Gohar Jehan²
}

\begin{abstract}
Objective: To determine the diagnostic accuracy of Doppler ultrasonography in predicting presence of esophageal varices in patients with hepatitis- $C$ induced cirrhosis.

Study Design: Descriptive analytical study.

Place and Duration of Study: Department of Radiology, Jinnah Hospital, Lahore, from May 2016 to October 2016.

Methodology: Patients aged 15-70 years, who presented with cirrhosis and proved to be cirrhosis caused by Hepatitis-C of any gender and duration of disease more than 6 months, were included. Patients with hepatocelullar carcinoma or portal vein thrombosis, having received treatment for esophageal varices, and unwilling to undergo endoscopy were excluded. All the patients then underwent Doppler ultrasonography to calculate splenoportal index and findings were correlated with endoscopy findings.

Results: Out of 200 patients, 137 (68.50\%) were males and 63 (31.50\%) were females. In Doppler ultrasonography positive patients, 113 were true positive and 8 were false positive. Overall sensitivity, specificity, positive predictive value, negative predictive value and diagnostic accuracy of Doppler ultrasonography in predicting presence of esophageal varices was $88.98 \%, 89.04 \%, 93.00 \%, 82.28 \%$ and $89.00 \%$, respectively.

Conclusion: Doppler ultrasonography is the non-invasive modality of choice with high diagnostic accuracy in predicting presence of esophageal varices in hepatitis-C induced cirrhosis.
\end{abstract}

Key Words: Doppler ultrasonography, Esophageal varices, Cirrhosis, Splenoportal index.

\section{INTRODUCTION}

Cirrhosis was the tenth leading cause of death in lowermiddle income countries in 2012.1 The leading cause of cirrhosis in Pakistan is hepatitis-C infection, accounting for $52-61 \%$ of cirrhosis cases. ${ }^{2}$ Out of every 100 people infected with HCV, between 5 and 20 develop cirrhosis.

Esophageal varix formation, resulting from portal hypertension, is a common major complication of cirrhosis and the most common clinical manifestation. ${ }^{3}$ Variceal development starts when hepatic venous pressure gradient reaches $10 \mathrm{mmHg} .{ }^{4} \mathrm{De}$ novo formation of varices occurs at a rate of $7-8 \%$ per year. 5 In Pakistan, the prevalence of esophageal varices is $65 \% .^{2}$

Presence of varices correlates with the severity of liver disease $-40 \%$ of patients with compensated and $60 \%$ with decompensated cirrhosis have esophageal varices. ${ }^{6}$ Varices develop and increase in size before eventually rupturing and bleeding, occurring in upto $30 \%$ of cirrhotic patients, ${ }^{7}$ and associated with a mortality rate of $16-24 \% .{ }^{8}$ Infact, the mortality rate from the first episode

1 Department of Radiology, Mayo Hospital, Lahore, Pakistan

2 Department of Radiology, Jinnah Hospital, Lahore, Pakistan

Correspondence: Dr. Ali Mansoor, Department of Radiology, Mayo Hospital, Lahore, Pakistan

E-mail:dr.alimansoor@hotmail.com

Received: August 28, 2018; Revised: December 24, 2018;

Accepted: December 24, 2018 of bleed is alarmingly high at $40-60 \%$ and only $30-40 \%$ of patients who do not receive treatment survive 2 years after the initiation of bleeding. ${ }^{9}$ Therefore, early detection of esophageal varices is imperative for reducing morbidity and mortality.

Endoscopy remains the gold standard for the diagnosis of esophageal varices and is also used therapeutically. Screening endoscopy for varices is recommended in all patients with cirrhosis. However, these recommendations are not a result of evidence-based data. 10 It has not yet been proven to be cost-effective for patients with compensated cirrhosis. ${ }^{11}$

Doppler ultrasonography has been suggested as an ideal tool for diagnosing portal hypertension. ${ }^{12} \mathrm{~A}$ number of studies have been conducted to assess the use of various Doppler ultrasonography indices for prediction of esophageal varices with conflicting results.13,14 Potentially useful indices studied include spleno-portal index,6,10 hepatic congestion index, and portal vein velocity. ${ }^{13}$

There is a dearth of local data available on this subject, with only one study, 15 done in Pakistani population regarding the use of Doppler ultrasonography for predicting esophageal varices in cirrhotic patients. Therefore, this study would add a non-invasive alternate for detecting complications of a relatively common disease in local population. The study was conducted to determine the accuracy of ultrasound indices in 
predicting the presence or absence of esophageal varices in patients with hepatitis- $C$ induced liver cirrhosis.

\section{METHODOLOGY}

This cross-sectional study was conducted in Department of Radiology, Jinnah Hospital, Lahore, from May 2016 to October 2016. Sample size of 200 was calculated with $95 \%$ confidence level taking prevalence of esophageal varices $65 \%, 5$ sensitivity $92 \%, 8$ margin of error $5 \%$, and specificity $93 \%, 8$ with margin of error $5.5 \%$ of Doppler ultrasonography in predicting presence of esophageal varices taking endoscopy as gold standard. Patients of either gender, aged 15 to 70 years, diagnosed with hepatitis-C induced cirrhosis for six months were included in the study through non-probability consecutive sampling. Patients having hepatocellular carcinoma, undergone treatment for esophageal varices, and having portal vein thrombosis were excluded.

After taking informed consent and relevant history, Doppler ultrasonography was performed in every patient using Color Doppler unit by a radiologist having 5 years' experience in Doppler sonography and blinded to the results of endoscopy to prevent observer bias. Grayscale ultrasonography was used to measure splenic dimensions and Doppler ultrasonography for portal vein velocity. These were then used to calculate the splenoportal index by the formula spleno-portal index= (splenic index / mean portal vein velocity). A value of $\geq 3.0$ was taken as presence of esophageal varices.

Endoscopy was then performed by gastroenterologist having over 5 years experience in endoscopy and blinded to the results of ultrasonography to remove observer bias. The spleno-portal index was then correlated with endoscopy findings by a third person.

Collected data were analysed through computer software SPSS 20.0. Mean and standard deviations were calculated for quantitative variables, i.e. age. Frequency and percentage was calculated for qualitative variables i.e. gender and presence or absence of esophageal varices on ultrasonography and endoscopy. Frequency table of $2 \times 2$ was used to calculate sensitivity, specificity, positive predictive value, negative predictive value and accuracy of Doppler ultrasonography in predicting presence of esophageal varices taking endoscopy as gold standard.

\section{RESULTS}

Age range in this study was from 21-69 years with mean age of $50.61 \pm 11.26$ years with majority of the patients $59(29.50 \%)$ between 50 to 60 years. Out of these 200 patients, $137(68.50 \%)$ were males and $63(31.50 \%)$ were females with male to female ratio of $2.17: 1$. Mean duration of disease was $27.92 \pm 15.23$ months.

All the patients were subjected first to Doppler ultrasonography to calculate splenoportal index and then
Table I: Summary of results.

\begin{tabular}{l|c|c|c}
\hline & $\begin{array}{c}\text { Positive results on } \\
\text { Doppler ultrasonography }\end{array}$ & $\begin{array}{c}\text { Negative results on } \\
\text { Doppler ultrasonography }\end{array}$ & \\
\hline $\begin{array}{l}\text { Positive results } \\
\text { on Endoscopy }\end{array}$ & 113 & 08 & \\
\hline Negative results & (True positive) & (False negative) & 121 \\
on Endoscopy & 14 & 65 & \\
\hline & (False positive) & (True negative) & 79 \\
\hline
\end{tabular}

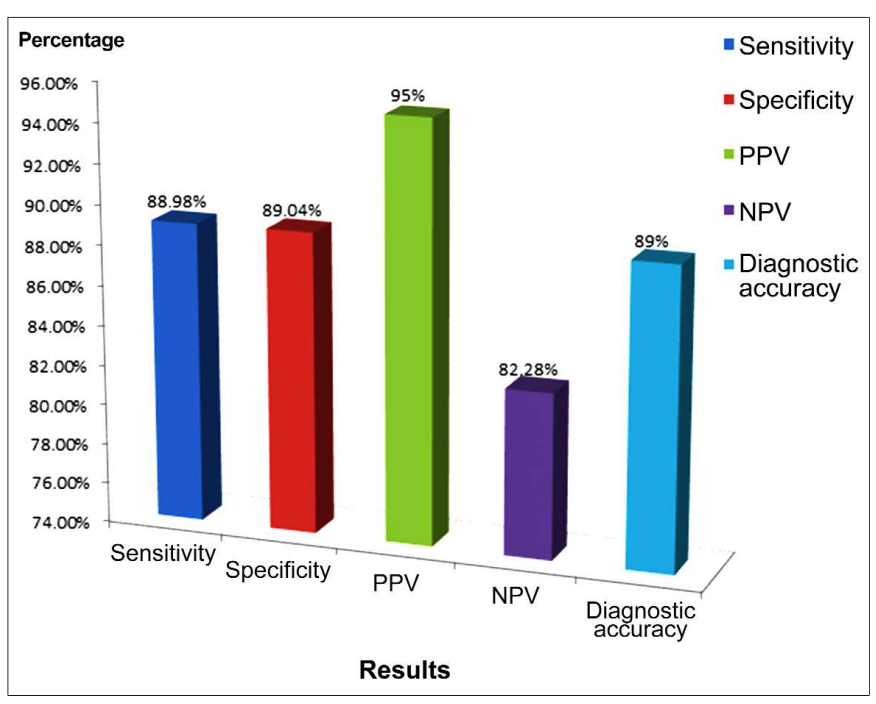

Figure 1: Diagnostic accuracy of Doppler ultrasonography in esophageal varices.

underwent endoscopy. Doppler ultrasonography findings favoured the presence of esophageal varices in 121 (60.5\%) patients. Endoscopy confirmed esophageal varices in 127 (63.5\%) patients; whereas, 73 (36.5\%) patients did not have esophageal varices. Among patients in whom Doppler ultrasonography findings supported the presence of esophageal varices, 113 (true positive) had varices and 8 (false positive) did not have varices on endoscopy. In those 79 patients in whom Doppler ultrasound findings did not favour presence of esophageal varices, 14 (false negative) had esophageal varices on endoscopy; whereas, 65 (true negative) did not have varices as shown in Table I.

Overall sensitivity, specificity, positive predictive value, negative predictive value and diagnostic accuracy of Doppler ultrasonography in predicting presence of esophageal varices was $88.98 \%, 89.04 \%, 93.00 \%$, $82.28 \%$ and $89.00 \%$, respectively (Figure I).

\section{DISCUSSION}

Esophageal varices tend to be a common complication of cirrhosis, estimated to occur in upto $50-61 \%$ of these patients. ${ }^{16}$ Although such patients can have bleeding episodes due to various causes, hematemesis due to these varices is the foremost among them. This bleeding can be life-threatening and once it occurs, chances of another episode increase dramatically; the rate of rebleeding has been reported to be as high as $70 \%$ with 
one-third of such episodes proving to be fatal. Therefore, the interest in developing a tool for early diagnosis of esophageal varices comes as no surprise.

Upper GI endoscopy is currently regarded as the gold standard for diagnosis of esophageal varices. Therefore, periodic screening endoscopy is currently recommended to all patients with established cirrhosis. ${ }^{3}$ Endoscopy not only serves as a diagnostic tool but also serves therapeutic options in the form of sclerotherapy and band ligation. However, many patients screened for esophageal varices do not have them. Therefore, endoscopy in these patients carries a useless burden of stress and financial burden on patients as well as unnecessary burden on health facilities. ${ }^{17}$ Moreover, the invasive nature of the process leads to low compliance, ${ }^{18}$ and can contribute to infections due to disruption of normal barriers. ${ }^{19} \mathrm{~A}$ non-invasive tool, which can serve the same purpose, is therefore, the need of the hour.

A number of biochemical and imaging parameters have been studied till date, to predict the presence of esophageal varices in cirrhotic patients. 20 Most of the studied imaging parameters utilise ultrasonographically measured indices such as splenic bipolar diameter, portal vein diameter, splenic vein diameter, spleno-portal index, hepatic congestion index, portal vein velocity and resistive indices of hepatic artery, splenic artery, renal artery, and superior mesenteric artery. In fact, ultrasonography offers a number of advantages, it is noninvasive, inexpensive and widely available. In this study, the diagnostic accuracy of one of the ultrasonographically determined indices, i.e. spleno-portal index was assessed for predicting the presence of esophageal varices in patients having cirrhosis induced by hepatitis- $\mathrm{C}$, taking endoscopy as gold standard.

In this study, sensitivity, specificity, positive predictive value, negative predictive value and diagnostic accuracy of Doppler ultrasonography in predicting presence of esophageal varices were $88.98 \%, 89.04 \%, 95.00 \%$, $82.28 \%$ and $89.00 \%$, respectively. In another study conducted by Liu et al., 10 a number of parameters were evaluated for predicting the presence of esophageal varices cirrhosis. The study concluded that spleno-portal index was the best among these parameters having sensitivity of $92 \%$, specificity of $93 \%$, positive predictive value of $91 \%$, and negative predictive value of $94 \%$ for esophageal varices. They reported a high diagnostic accuracy of $92-94 \%$ for this index.

A similar study was conducted locally in province of Sindh which studied splenoportal index as well as platelet count to splenic diameter ratio for similar purposes. ${ }^{15}$ This study reported sensitivity, specificity, positive predictive value, negative predictive value and diagnostic accuracy of $75 \%, 65.1 \%, 77.3 \%, 62.2 \%$ and $71.2 \%$, respectively of spleno-portal index for diagnosing esophageal varices.
The pathophysiologic changes that accompany the process of cirrhosis could explain the usefulness of splenoportal index in predicting esophageal varix formation. Fibrosis in liver leads to increased portal resistance. This leads to stagnation of portal blood flow resulting in increased resistance to venous outflow from spleen. Consequently, congestive splenomegaly develops. Concomitantly, increased splanchnic inflow augments the splenomegaly and aggravates portal hypertension. The increase in portal hypertension facilitates esophageal varix formation as well as further augmenting the splenomegaly. 21 It is not surprising, therefore, that splenomegaly is common in cirrhosis. ${ }^{22}$ Previous studies have also shown that decrease in mean portal vein velocity correlated with severity of portal hypertension and risk of bleeding from esophageal varices. ${ }^{23}$ Splenoportal index incorporates both these parameters, i.e. splenic size as well as portal vein velocity. Therefore, it has been shown to be a useful non-invasive tool for diagnosis of esophageal varices.

In addition to its non-invasive nature, splenoportal index offers a couple of other advantages over other parameters. First, it can be concomitantly measured during routine biannual ultrasound screening for hepatocellular carcinoma in cirrhotic patients; hence, reducing additional costs. Secondly, it can be measured easily in outpatient setting unlike other biochemical tests and endocopy. So, it is recommended that Doppler ultrasonography should be routinely used for predicting presence of esophageal varices in cirrhosis rather than subjecting them to invasive endoscopy which should be reserved for therapeutic interventions.

This study differed from other similar studies in that the study population comprised of a relatively homogenous group consisting of patients having cirrhosis induced by chronic infection with hepatitis- $C$ virus. While most of the subjects in the other studies also had hepatitis-C induced cirrhosis, there were subjects with cirrhosis due to other etiologies as well.

\section{CONCLUSION}

This study concluded that Doppler ultrasonography is the non-invasive modality of choice with high diagnostic accuracy in predicting presence of esophageal varices in patients with Hepatitis-C induced cirrhosis. It can help reduce the financial burden on health facilities as well as better compliance of patients as compared to endoscopy. These factors coupled with wider availability and requirement of lesser expertise further highlight its emerging role for this purpose.

\section{REFERENCES}

1. World Health Organisation. The top 10 causes of death [Internet]. 2016. [cited 2018 Aug 18] Available from:http:// www.who.int /mediacentre/factsheets/fs310/en/index1.html 
2. Ullah F, Khan S, Afridi AK, Rahman SU. Frequency of different causes of cirrhosis liver in local population. Gomal J Med Sci 2012; 10:178-81.

3. Shehata M, AboAli LA, El-Shafey K, El-Hossary M. A comparative study of duplex doppler ultrasound and blood indices as noninvasive predictors of oesophageal varices in cirrhotic patients. Tanta Med J 2014; 42:83-91.

4. Rezayat KA, Ghanaei FM, Alizadeh A, Shafaghi A, Jandaghi AB. Doppler surrogate endoscopy for screening esophageal varices in patients with cirrhosis. Hepatitis Monthly 2014; 14.

5. Bintintan A, Chira RI, Bintintan W, Nagy GA, Manzat-Saplacan MR, Lupsor-Platon $\mathrm{M}$, et al. Value of hepatic elastography and doppler indexes for predictions of esophageal varices in liver cirrhosis. Medical Ultrason 2015; 17:5-11

6. Bintintan A, Chira RI, Mircea PA. Non-invasive ultrasound based diagnosis and staging of oesophageal varices in liver cirrhosis: A systematic review of literature published in the third millennium. Med Ultrason 2013; 15:116-24.

7. Nada L, El Fakir S, Bahija B, Adil I, Nourdine A. Noninvasive predictors of presence and grade of esophageal varices in viral cirrhotic patients. Pan Afr Med J 2015; 20:145.

8. Reverter E, Tandon P, Augustin S, Turon F, Casu S, Bastiampillai $\mathrm{R}$, et al. A MELD-based model to determine risk of mortality among patients with acute variceal bleeding. Gastroenterol 2014; 146:412-9.

9. Giannini E, Botta F, Borro P, Risso D, Romagnoli P, Fasoli A, et al. Platelet count/spleen diameter ratio: Proposal and validation of noninvasive parameter to predict the presence of oesophageal varices in patients with liver cirrhosis. Gut 2003; 52:1200-5.

10. Liu CH, Hsu SJ, Liang CC, Tsai FC, Lin JW, Liu CJ, et al. Esophageal varices: Noninvasive diagnosis with duplex doppler US in patients with compensated cirrhosis. Radiol 2008; 248:132-9.

11. Arguedas MR, Heudebert GR, Eloubeidi MA, Abrams GA, Fallon MB. Cost-effectiveness of screening, surveillance, and primary prophylaxis strategies for esophageal varices. $A m \mathrm{~J}$ Gastroenterol 2002; 97:2441-52.

12. Keerthi K, Kumar HS, Kalra VB, Kusumalatha P, Sunil A. Doppler - a very helpful diagnostic tool for evaluating portal hypertension. J Evid Based Med Health 2015; 2:2220-24.
13. Shastri M, Kulkarni S, Patell R, Jasdanwala S. Portal Vein doppler: A tool for non-Invasive prediction of esophageal varices in cirrhosis. J Clin Diagn Res 2014; 8:MC12-5.

14. Jalli R, Hosseini-AsI MK, Eshraghian A, Rezaei B. Ultrasound based techniques and transient elastography may not be precise methods for the detection of esophageal varices in liver cirrhosis. Med Ultrason 2014; 16:78-9.

15. Wadhwa RK, Abbas Z, Mujahid Hasan S, Luck NH, Younus M, Anis $\mathrm{S}$, et al. Platelet count to splenic diameter ratio and splenoportal index as non-invasive screening tools in predicting esophageal varices in patients with liver cirrhosis. J Translat Intern Med 2014; 2:127-31.

16. Thomopoulos KC, Labropoulou-Karatza C, Mimidis KP, Katsakoulis EC, Iconomou G, Nikolopoulou VN. Non-invasive predictors of the presence of large oesophageal varices in patients with cirrhosis. Dig Liver Dis 2003; 35:473-8.

17. de Franchis R. Revising consensus in portal hypertension: report of the Baveno $\mathrm{V}$ consensus workshop on methodology of diagnosis and therapy in portal hypertension. J Hepatol 2010; 53:762-8.

18. De Franchis R. Non-invasive (and minimally invasive) diagnosis of oesophageal varices. J Hepatol 2008; 49:520-7.

19. Khalil FA, Khalil KA, Khalil TH, Hassan AA, Serwah MA. Evaluation of clinical, biochemical and ultrasound parameters in diagnosis of oesophageal varices. Med J Cairo Uni 2010; 78:105-9.

20. Gill ML, Atiq M, Sattar S, Khokhar N. Non-endoscopic parameters for the identification of esophageal varices in patients with chronic hepatitis. J Pak Med Assoc 2004; 54:575-7.

21. Watanabe S, Hosomi N, Kitade Y, Kurokohchi K, Arima K, Kawabata $\mathrm{H}$, et al. Assessment of the presence and severity of esophagogastric varices by splenic index in patients with liver cirrhosis. J Comput Assist Tomogr 2000; 24:788-94.

22. Bolognesi M, Merkel C, Sacerdoti D, Nava V, Gatta A. Role of spleen enlargement in cirrhosis with portal hypertension. Dig Liver Dis 2002; 34:144-50.

23. Taourel P, Blanc P, Dauzat M, Chabre M, Pradel J, Gallix B, et al. Doppler study of mesenteric, hepatic, and portal circulation in alcoholic cirrhosis: Relationship between quantitative Doppler measurements and the severity of portal hypertension and hepatic failure. Hepatology 1998; 28:932-6. 